\title{
In Silico Screening of Sesquiterpene Lactones as Aldose Reductase Inhibitors
}

\author{
${ }^{*}$ A.M. Alhassan and 2I. Malami \\ 1Department of Pharmaceutical and Medicinal Chemistry, Faculty of Pharmaceutical Sciences, Usmanu Danfodiyo University, Sokoto, \\ Nigeria \\ 2Department of Pharmacognosy and Ethnobotany, Faculty of Pharmaceutical Sciences, Usmanu Danfodiyo University, Sokoto, \\ Nigeria \\ [*Corresponding Author: E-mail: alhasanma@yahoo.com; D: +2348139766462]
}

\section{ABSTRACT}

Aldose reductase, a key enzyme of the polyol pathway catalyses NADPH-dependent reduction of glucose to sorbitol. Increased activity of this enzyme is considered a major factor contributing to the development of diabetic complications hence could be an important target in the treatment of these complications. In this work, a database of sesquiterpenes was prepared and screened for their drug-like properties based on the Lipinski's rule of 5. The co-crystallised structure of aldose reductase was obtained from the Protein Data bank and prepared for docking. In silico docking experiments was performed on Autodock tools using 198 sesquiterpene lactones that passed screening, and compounds with the lowest binding energy and favourable binding interactions were selected for molecular docking simulation. Six of the best ranking compounds selected had binding energies ranging from-11.96 Kcal/mol to $-9.45 \mathrm{Kcal} / \mathrm{mol}$ and were comparable to the energy of the standard inhibitor Idd594 used in the study. They also show good complementarity in their binding to the residues of the binding pocket. The results suggest that dehydrooopodin (1), 11(S),13-dihydrolactucopicrin (2), and Chrysanin (3) offered potential inhibitory activities toward aldose reductase and may serve as lead compounds for in vivo validation as aldose reductase inhibitors.

Keywords: Sesquiterpene lactones, Aldose reductase, Binding energy, Molecular docking, Autodock

\section{INTRODUCTION}

Diabetes mellitus (DM) is a group of endocrine metabolic dysfunctions characterized by an abnormal increase in blood glucose levels resulting from inadequate insulin production, decreased insulin sensitivity and function, or excessive glucagon secretion (Blair, 2016; Tan et al., 2019). The global burden of diabetes mellitus is very high and is increasing rapidly. According to the International Diabetic Federation (IDF), it is estimated that 451 million people were living with diabetes in 2017 and the prevalence is expected to reach 693 million by 2045 (IDF, 2017). Diabetes is responsible for an estimated global economic expense costing about US\$727 billion of the total global adult health expenditure in 2017 (Afroz et al., 2019), and a projected increased is expected to be US\$776 billion by 2045 (IDF, 2017).
The most devastating effect of chronic DM is the gradual progressive damage of vascular endothelial cells, which result in diabetic complications (Georgescu, 2011). Retinopathy, among the chronic complications of diabetes, is the leading cause of blindness (Garofolo et al., 2019; Hippisley-Cox and Coupland, 2016). Evidence from biochemical studies has shown that the polyol pathway plays a major role in the development of retinopathy (Oates, 2008; Tang et al., 2012). Aldose reductase (AR2, EC1.1.1.21) is the first enzyme of the polyol pathway. It catalyses the reduction of glucose to sorbitol with concomitant oxidation of NADPH to NADP+. Increased activity of this enzyme causes accumulation of sorbitol leading to osmotic stress. In addition, the depletion of NADPH causes alterations in cellular redox potentials and reduced activity of enzymes such as nitric oxide synthase (NOS) and glutathione reductase which leads to cellular oxidative stress (Forbes and 


\section{Alhassan and Malami: In Silico Screening of Sesquiterpene Lactones as Aldose Reductase.....}

Cooper, 2013). The osmotic and oxidative stress contribute to cellular damage through the activation of extracellular signal transduction cascade and subsequent expression of inflammatory cytokines (Kashihara et al., 2010; Tang et al., 2012; Zehra and Zarina, 2012).

Aldose reductase has been targeted for the treatment of diabetic complications. Inhibitors of this enzyme have been shown to delay the onset and slow the progression to chronic diabetic complications in experimental animal models (Chung and Chung, 2005). Unfortunately, most of the aldose reductase inhibitors (ARIs) developed over the past three decades fail to pass the phase III clinical trials either due to toxicity or pharmacokinetic problems (Chung and Chung, 2005). At present, only one aldose reductase inhibitor-Epalrestat is commercially available (Sato et al., 2014). Hence, there is need for further research into newer ARIs. Molecular docking techniques have been used to study molecules that are likely to bind to a drug target in silico. The autodock suite is one of several softwares that has been developed for automated docking programs designed to predict how small molecules bind to 3D structures of target receptors. Besides calculating the binding energies, Autodock tools have graphical user interphase (GUI) that can be used to visualize the binding mode of the ligands, which is very useful in drug design. This technique is explored in this study to characterize sesquiterpene lactones group of secondary metabolites commonly found in plants belonging to the family, Astraceae (Ivanescu et al., 2015) for their possible inhibitory effects on aldose reductase enzyme in silico.

\section{MATERIALS AND METHODS Software and Programs}

The virtual screening of sesquiterpene lactones was carried out in a Linux cluster system in Pharmaceutical Design and Simulation laboratory, Universiti Sains Malaysia. The programs that were used include Python 2.5 available at www.python.com, Molecular Graphics library MGLTools1.5.6, AutodockTools
4.2 (for in silico docking experiments), and Racconautodock available at www.scripps.edu. Other programs used include Discovery Studio 2.2.5, Marvinsketch (Chemaxon), and Microsoft Excel (version, 2013).

\section{Preparation of Ligand Database}

A ligand database of 208 sesquiterpene lactones was obtained from Pubchem, Chemspider and Zinc databases and saved in sdf format. Few of the compounds obtained from literature and their chemical structures were built using the Marvinsketch (http://www.chemaxon.com/mychemaxon/my-academic-license/). The prepared ligand program Discovery studio 2.5.5 (Accelrys Software Inc.) was used for geometry optimization and subsequent screening of the ligands based on Lipinski's rule (Christiphor et al., 2019). A total of 198 ligands that passed the Lipinski's rule were converted to pdbqt file format using a Raccoon Autodock program (Forli et al., 2016) and saved for virtual screening.

\section{Aldose Reductase Docking Simulation Studies}

A high-resolution X-ray diffraction structure of human aldose reductase in complex with known inhibitor Idd594 (PDB code: 1US0) was retrieved from Protein Data Bank. The macromolecule was prepared using Autodock 4.2 (Morris et al., 2009). Ligand and water molecules were removed, all hydrogens were added, and Gasteiger charges were calculated for each of the molecules. The prepared macromolecules were saved in pdbqt format. To ensure the correct conformation of ligand binding to active sites during virtual screening, a grid parameter file (GPF) was generated for the receptor and its co-crystallised ligand by identifying Coordinates of Central Grid Point of Maps (GridBox). Subsequently, docking validation was performed by re-docking the cocrystallized ligands Idd594 on binding sites of the aldose reductase receptor. The rotational bonds of the ligands were treated as flexible, while those of the protein were kept rigid. Grid boxes were fixed around the NADPH -binding site using the ligand as the grid box centre. The box size 
was set to 50,50 , and $60 \AA 3(x, y$, and $z$, respectively) and the grid spacing to $0.375 \AA$. The grid maps for atom were calculated for each ligand types for each of the three ligands. Genetic Algorithm (GA) was used for searching, and the population size was set to 150,100 runs, and 5 million energy evaluations. The grid and docking parameters were then computed for all ligands using parameters obtained from the re-docking of co-crystallized ligands as reference. The intermolecular interactions were computed as free binding energies as follows:

Free Binding Energy $=P+Q+R-S$ (Huey et al., 2007)

where, $P$ represents final intermolecular energy + van der Walls energy $(v d W)+$ hydrogen bonds + desolvation energy + electrostatic energy $(\mathrm{kcal} / \mathrm{mol}) ; \mathrm{Q}$ represents final total internal energy $(\mathrm{kcal} / \mathrm{mol}) ; \mathrm{R}$ represents torsional free energy $(\mathrm{kcal} / \mathrm{mol}) ; \mathrm{D}$ represent unbound system's energy (kcal/mol).

\section{RESULTS}

Table 1 shows zero Lipinski rule violations for six selected compounds: dehydrooopodin (1), 11(S),13-dihydrolactucopicrin (2), Chrysanin (3), Subcordatolide C (4), Budlein A (5), and Vernodalin (6), which is an indication of favourable bioavailability of selected molecules (Figure 1).

Table 1: Properties of compounds selected top ranked compounds

\begin{tabular}{clllll}
\hline Compounds & $\mathrm{Mw}$ & nHBA & nHBD & LogP & PSA(Å2) \\
\hline $\mathbf{1}$ & 328.4 & 2 & 0 & 4.21 & 56.60 \\
$\mathbf{2}$ & 412.4 & 5 & 2 & 1.81 & 110.13 \\
$\mathbf{3}$ & 356.5 & 3 & 1 & 2.28 & 72.83 \\
$\mathbf{4}$ & 334.4 & 3 & 1 & 2.75 & 72.83 \\
$\mathbf{5}$ & 372.4 & 5 & 1 & 1.88 & 99.13 \\
$\mathbf{6}$ & 360.7 & 4 & 1 & 1.60 & 99.13 \\
\hline
\end{tabular}

$\mathrm{Mw}=$ molecular weight, $\mathrm{nHBA}=$ number of hydrogen bond, $\mathrm{nHBD}=$ nunber of hydrogen bond donors, $\mathrm{PSA}=$ polar suface area

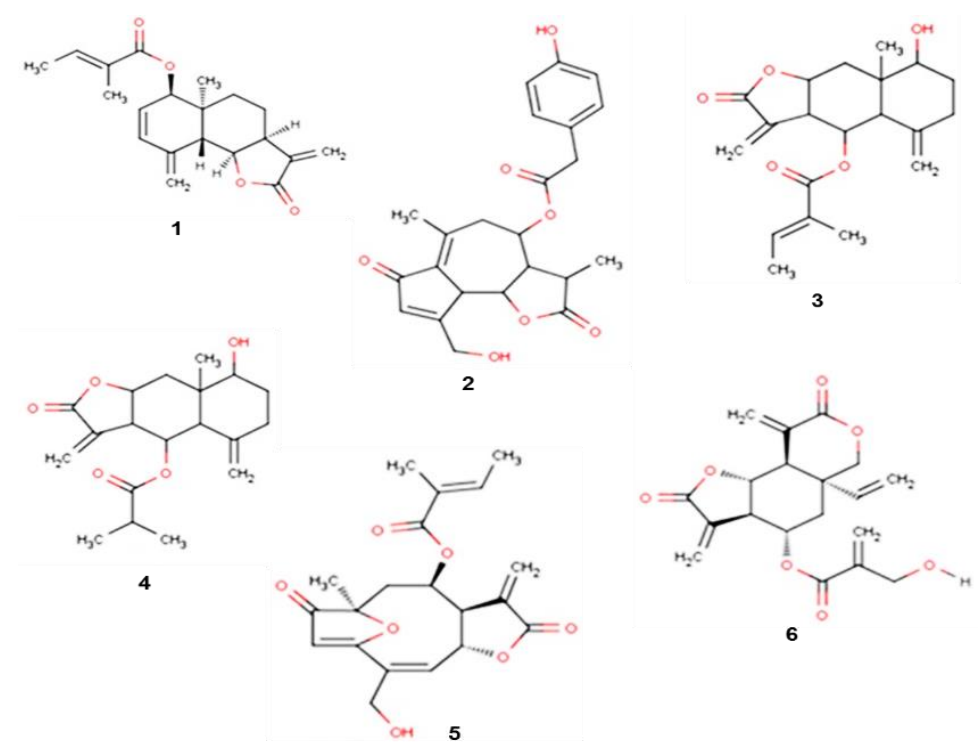

Figure 1: Chemical structure of selected compounds 1-6. 
The amino acid sequence Trp20, Val 47 Tyr48, Trp79, His 110, Trp111, Phe122 Trp219, Cys298, Ala299, and Leu300 were defined in the binding site of the crystallized ligand Idd594 (Figure 2A and $B$ ). The active site comprised two different pockets, the catalytic pocket, and specificity pocket (Figure 2A). The catalytic pocket is deeply buried and is composed of polar residues (Tyr48,
Lys77, His110, Trp111), nicotinamide moiety of the co-factor NADPH, and the other hydrophobic residues constitute the specificity pocket. The redocking of reference ligand (Idd594) into the active site of ALR2 occupies the same binding pocket at root mean square deviation (RMSD) of $0.88 \mathrm{~A}$, which further validates the present docking protocol (Figure 2B).

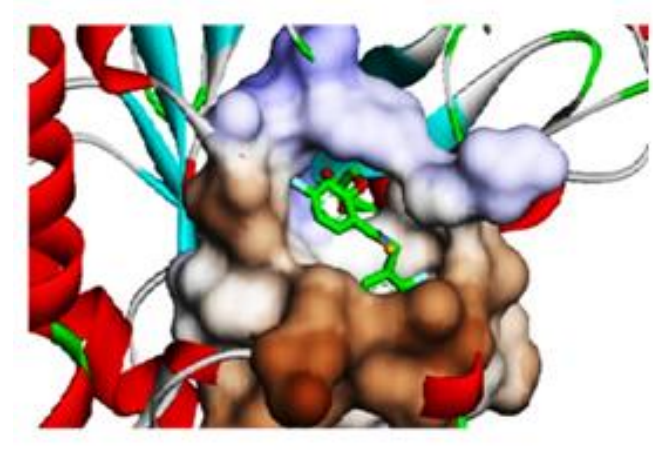

A

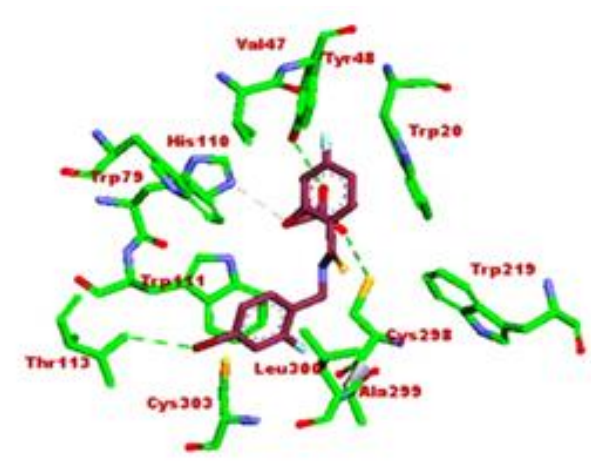

B

Figure 2: Active site of ALR2 - (A) Surface structure of the active site with 1dd594. Ligand is shown in green, hydrophobic sub-pocket in brown and anionic sub-pocket region in blue. (B) Crystal pose of idd594 interacting with amino acid residues. Ligand is shown in magenta colour, Hydrogen bonds in green dotted lines.

Table 2 shows the ranking of the molecular binding energies of compounds $1-6$ which range from $-11.5 \mathrm{kcal} / \mathrm{mole}$ to $-9.45 \mathrm{kcal} / \mathrm{mol}$. The compound with the lowest energy is expected to have the highest inhibition constant. Compounds 1-3 have lower binding energies when compared with the standard ligand idd594 $(-10.55 \mathrm{kcal} / \mathrm{mol})$ which suggest better inhibition constant while compounds 4-6 have lesser binding affinity than the co-crystallized reference ligand. This is an indication that the selected compounds demonstrate a good binding affinity for ALR2.

Figure $3(A-E)$ shows the binding poses of the six compounds with the amino acid residues of the ALR2 active site. In docking pose of compound 1
(Figure 3A), the ester group of the acrylate side chain is positioned in the catalytic pocket were the carbonyl oxygen form hydrogen bonding with Tyr48, His110, and Trp111, the other ring portion form hydrophobic interaction with non-polar residues. The carbonyl oxygen of the $y$-lactone ring form additional $\mathrm{H}$-bonding with backbone oxygen of Leu300 and Ala299. Compounds 2 - 6 display a common binding pattern, different from the binding mode seen in 1 (Figure 3B-F). The carbonyl group of the $y$-lactone of these compounds is positioned in the catalytic pocket where it exhibits hydrogen bonding with polar Tyr48, His110, and/or Trp111. The other cyclic portion and side-chain form hydrophobic interactions with non-polar residues. 
Nigerian Journal of Basic and Applied Science (December, 2020), 28(2): 64-69

Table 2: Binding energy, hydrogen bond and hydrophobic interactions of the six selected ligands

\begin{tabular}{|c|c|c|c|c|}
\hline COMPOUND & $\begin{array}{l}\text { BINDING } \\
\text { ENERGY } \\
\text { (KCAL/M } \\
\text { OL) }\end{array}$ & $\begin{array}{l}\text { HYDROGEN } \\
\text { BOND } \\
\text { INTERACTIONS }\end{array}$ & $\begin{array}{l}\text { HYDROPHOBIC } \\
\text { INTERACTIONS }\end{array}$ & $\begin{array}{l}\text { NUMBER OF AMINO } \\
\text { ACID RESIDUES } \\
\text { INTERACTING WITH } \\
\text { THE LIGAND }\end{array}$ \\
\hline \multirow[t]{2}{*}{1} & -11.96 & $\begin{array}{ll}\text { Tyr48, } & \text { His110, } \\
\text { Leu } 300 & \end{array}$ & $\begin{array}{l}\text { Trp20, Trp111, Trp219, Cys } \\
\text { 298, Leu300 }\end{array}$ & 8 \\
\hline & -10.57 & Try48, His110 & $\begin{array}{l}\text { Trp79, Trp111, Cys298, } \\
\text { Leu300, Cys303 }\end{array}$ & 7 \\
\hline 3 & -10.56 & Try48, His110 & $\begin{array}{l}\text { Trp20, Val47, Trp111, } \\
\text { Trp219, Cys 298, Leu300 }\end{array}$ & 8 \\
\hline 4 & -9.97 & $\begin{array}{l}\text { Try48, His110, } \\
\text { Trp111 }\end{array}$ & $\begin{array}{l}\text { Trp20, Val47, Trp219, Cys } \\
298\end{array}$ & 7 \\
\hline 5 & -9.88 & $\begin{array}{l}\text { Tyr48, His110, } \\
\text { Trp111 }\end{array}$ & Trp20, Cys 298 & 5 \\
\hline 6 & -9.45 & $\begin{array}{l}\text { Tyr48, His 110, } \\
\text { Ala } 299\end{array}$ & $\begin{array}{l}\text { Trp20, Trp111, Trp219, Cys } \\
298\end{array}$ & 6 \\
\hline $\begin{array}{l}\text { Idd594 (Co- } \\
\text { crystalized } \\
\text { ligand) }\end{array}$ & -10.55 & $\begin{array}{l}\text { Tyr48, His110, } \\
\text { Cys 298, Thr113 }\end{array}$ & None & 4 \\
\hline
\end{tabular}
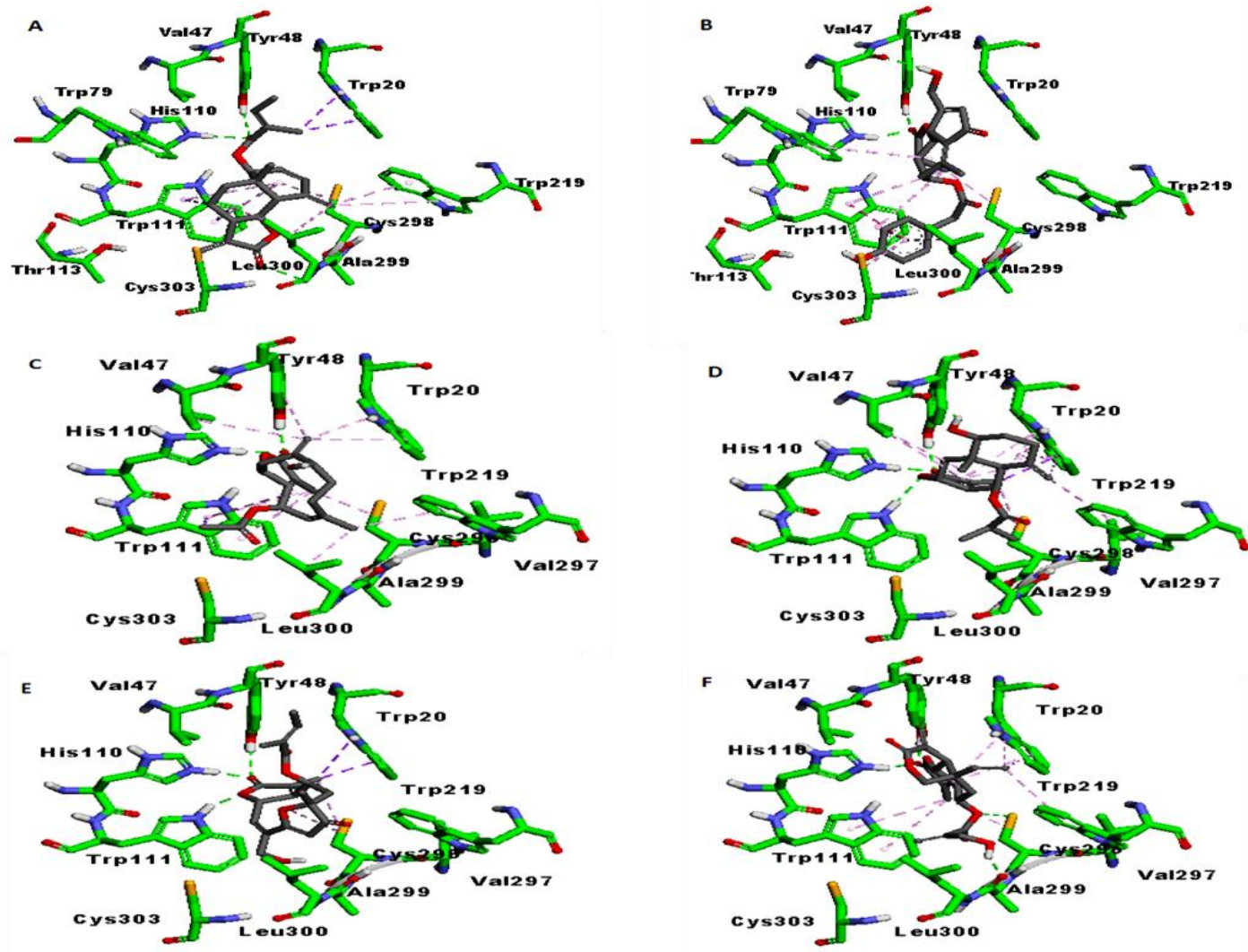

Figure 3: Docking poses of ligands interactions with amino acid residues. A - F poses of compounds $1-6$, respectively. Ligands are shown in grey colour, hydrogen bond in green dotted lines, hydrophobic interactions in purple dotted lines. 


\section{DISCUSSION}

The amino acid sequence Trp20, Val Tyr48, Trp79, His 110, Trp111, Phe122 Trp219, Cys298, Ala299, and Leu300 that defines the active site of aldose reductase formed an intermolecular interaction with the sesquiterpene lactones. As a general rule, hydrogen bond interaction with the Tyr48, His110, and Trp111 in the catalytic pocket and hydrophobic interactions with polar residues of the specificity pocket have been found to be responsible for mediating the aldose reductase inhibition (El-Kabbani et al., 2004; Howard et al., 2004). The binding modes for selected ligands were inspected and each ligand selected based on the criteria that it must exhibit hydrogen bonding with at least two of the residue Tyr48, His110 and Trp111, and must have hydrophobic interaction with relevant residues in the specificity pockets (Trp20, Trp111, Cys298, Ala299, and Leu300). This suggests that compounds $1-6$ demonstrate the potential to bind and inhibit the catalytic ability of ALR2.

The binding of the lactone group of sesquiterpene lactones into the catalytic pocket of aldose reductase observed in the present study has been similarly observed in the findings of Wang et al. (2008) where they observed coumarin lactone ring act as an acceptor to build the hydrogen bond network with the polar residues of the catalytic pocket of aldose reductase. Another study by Wang et al. (2009) showed the binding of the lactone ring of a group of phenolic marine compounds into the catalytic pocket of aldose reductase displayed high stability during the molecular dynamics studies. The central lactone ring was also shown to exhibit similar binding mode as revealed in this study. This is an indication that the lactone could serve as an alternative functional group to cyclic imide and carboxylic acid in the development of newer aldose reductase inhibitors.

\section{CONCLUSION}

Based on the docking study, the selected sesquiterpene lactones were found to have binding energies that stabilize their interaction with the target enzyme. Specifically, compounds 1,2 , and 3 had lower binding energies than the standard aldose reductase inhibitor (Idd594). Findings from the present study suggest that the selected sesquiterpene lactones offer a potential inhibitory activity towards aldose reductase enzyme and may be validated further through in vivo studies.

\section{REFERENCES}

Afroz, A., Alam, K., Ali, L., Karim, A., Alramadan, M.J., Habib, S.H., Magliano, D.J., \& Billah, B. (2019). Type 2 diabetes mellitus in Bangladesh: A prevalence based cost-ofillness study. BMC Health Services Research, 19: 601.

Blair, M. (2016). Diabetes Mellitus Review. Urologic Nursing, 36(1): 27-36.

Christiphor, A.L., Franco, L., Beryl, W.D., \& Paul, J.F. (2019). Experimental and computational approaches to estimate solubility and permeability in drug discovery and development settings. Advanced Drug Delivery Review, 23: 3-25.

Chung, S., Chung, S., 2005. Aldose Reductase in Diabetic Microvascular Complications. Current Drug Targets. 6, 475-486.

El-Kabbani, O., Darmanin, C., Schneider, T.R., Hazemann, I., Ruiz, F., Oka, M., Joachimiak, A., Schulze-Briese, C., Tomizaki, T., Mitschler, A., \& Podjarny, A. (2004). Ultrahigh resolution drug design. II. Atomic resolution structures of human aldose reductase holoenzyme complexed with fidarestat and minalrestat: Implications for the binding of cyclic imide inhibitors. Proteins, 55(4): 805-813. 